\title{
Significância clínica do uso do mapa mental na intervenção de escolares com transtornos de aprendizagem
}

\author{
Clinical significance of using the mental \\ map in interventions on schoolchildren with \\ learning disorders
}

\section{Significado clínico del uso del mapa mental en la intervención de estudiantes con transtornos de aprendizaje}

\author{
Lívia Nascimento Bueno* \\ Alexandra Beatriz Portes de Cerqueira César* \\ Bianca dos Santos* (ID \\ Simone Aparecida Capellini* (D)
}

\section{Resumo}

Introdução: Para um bom desempenho de leitura, o escolar precisa ser capaz de desenvolver habilidades específicas para compreensão da informação lida, criando estratégias como: construção de significado, assimilação do conteúdo, estabelecimento de relações, organização e avaliação das informações, bem como pensar na utilização dessas de forma correta. Objetivo: verificar o índice de mudança e significância clínica do Mapa Mental na intervenção de escolares com transtornos de aprendizagem em situação de pré e pós-testagem. Métodos: Este estudo se caracteriza por ser quaseexperimental. Participaram deste estudo 7 escolares com diagnóstico interdisciplinar de transtornos de aprendizagem, de ambos os sexos, na faixa etária de 10 anos e 6 meses a 15 anos e 4 meses de idade. Os escolares foram submetidos à pré-testagem, intervenção e pós-testagem. A pré-testagem e a póstestagem foram realizadas com o Protocolo de Avaliação de Compreensão de Leitura (PROCOMLE), e

* Faculdade de Filosofia e Ciências - UNESP - Campus de Marília, Marília, SP, Brasil.

Contribuição dos autores:

LNB: concepção do estudo; coleta de dados; metodologia. ABPCC: metodologia; esboço da discussão dos resultados. BS: análise estatística dos dados; descrição dos resultados.

SAC: orientação; metodologia; revisão crítica.

E-mail para correspondência: Lívia Nascimento Bueno - livianascbueno@gmail.com Recebido: $18 / 06 / 2020$

Aprovado: $11 / 06 / 2020$ 
a intervenção foi realizada com o uso do mapa mental durante 4 meses, sendo trabalhados 15 textos. Os dados de pré e pós-testagem foram analisados por meio do Método JT. Resultados: na comparação da pré com a pós-testagem, foi possível verificar que os escolares S1, S2, S3 e S6 apresentaram mudança confiável nas questões literais de textos narrativos do Protocolo de Avaliação de Compreensão de Leitura. Os escolares S1, S3, S6 e S7 apresentaram mudança confiável nas questões inferenciais de textos narrativos do Protocolo de Avaliação de Compreensão de Leitura. Conclusão: a intervenção com o uso do Mapa Mental mostrou-se eficaz para escolares com Transtornos de Aprendizagem.

Palavras-chave: Compreensão; Leitura; Estratégias; Aprendizagem.

\section{Abstract}

Introduction: For a good reading performance, the student needs to be able to develop specific skills to understand the information read, creating strategies such as: construction of meaning, assimilation of content, establishment of relationships, organization and evaluation of information, as well as thinking about using these correctly. Objective: to verify the rate of change and clinical significance of the Mind Map in the intervention of students with learning disorders in pre- and post-testing situations. Methods: This study is characterized as quasi-experimental. The pretest and post-test were done using the Reading Comprehension Evaluation Protocol (PROCOMLE), and the intervention was accomplished by applying mind mapping techniques on studies of 15 texts within 4 months. The pre and posttest data were analyzed using the JT Method. Results: Comparison of the pre- and post-testing, using the JT Method, showed that: students S1, S2, S3 and S6 presented a reliable change in the literal questions of narrative texts from the Reading Comprehension Assessment Protocol; and students S1, S3, S6 and S7 presented a reliable change in the inferential questions. Conclusion: The intervention using Mind Mapping proved to be effective for students with Learning Disorders.

Keywords: Comprehension; Reading; Strategies; Learning.

\section{Resumen}

Introducción: Para un buen desempeño de lectura, el estudiante debe ser capaz de desarrollar habilidades específicas para comprender la información leída, crear estrategias tales como: construcción de significado, asimilación de contenido, establecimiento de relaciones, organización y evaluación de información, así como pensar en úselos correctamente. Objetivo: verificar el índice de cambios y significado clínico del Mapa Mental en la intervención de estudiantes con trastornos de aprendizaje en situación de pre y post prueba. Métodos: Este estudio se caracteriza por ser cuasi-experimental. En este estudio participaron siete estudiantes con diagnóstico interdisciplinario de trastornos del aprendizaje, de ambos sexos, de 10 años y 6 meses a 15 años y 4 meses de edad. Los estudiantes fueron sometidos a pruebas previas, de intervención y posteriores. El pretest y el postest se realizaron mediante el Protocolo de Evaluación de Comprensión Lectora (PROCOMLE) y la intervención se realizó mediante un mapa mental durante 4 meses, trabajando en 15 textos. Los datos anteriores y posteriores a la prueba se analizaron mediante el método JT. Resultados: En la comparación de la pre con la post prueba, a partir del Método JT, ha sido posible comprobar que los estudiantes S1, S2, S3 y S6 han presentado cambios confiables en las preguntas literales de textos narrativos del Protocolo de Evaluación de Comprensión de Lectura. Los estudiantes S1, S3, S6 y S7 han presentado cambios confiables en las preguntas inferencias de textos narrativos del Protocolo de Evaluación de Comprensión de Lectura. Conclusión: La intervención con el uso del Mapa Mental resultó efectivo para estudiantes con Trastornos de Aprendizaje.

Palabras clave: Comprensión; Lectura; Estrategias; Aprendizaje. 


\section{Introdução}

Silva e Capellini ${ }^{1}$, e Silver e colaboradores ${ }^{2}$ definem os Transtornos de Aprendizagem como um grupo de dificuldades heterogêneas caracterizadas pela presença de uma disfunção neurológica, que é responsável pelo insucesso na escrita, na leitura e no cálculo matemático.

A leitura é uma atividade muito exigida em todas as fases de nossas vidas e também considerada muito complexa, sendo essencial para a compreensão de um texto, o que extrapola todo o seu conteúdo exposto. Além da compreensão do material lido, a leitura está relacionada com a aprendizagem no geral, pois está presente em todos os aspectos e métodos ensinados na escola; todas as disciplinas vão exigir que o escolar tenha uma fluência de leitura, como, por exemplo, para compreender um cálculo matemático o escolar deve ser capaz de compreender o enunciado e o que lhe foi pedido. Já no texto, o escolar deve ser capaz de entender os acontecimentos principais e saber relacionar todos eles, sempre reconhecendo o ciclo temporal do material lido ${ }^{3}$.

Para um bom desempenho de leitura, o escolar precisa ser capaz de desenvolver habilidades específicas para compreensão da informação lida, criando estratégias como: construção de significado, assimilação do conteúdo, estabelecimento de relações, organização e avaliação das informações, bem como pensar na utilização dessas de forma correta. Os escolares com transtornos de aprendizagem apresentam um desempenho inferior no desenvolvimento de tais habilidades, como também nas habilidades de escrita e matemática ${ }^{4,5,6,7}$.

Os escolares com transtornos de aprendizagem vão apresentar um desempenho inferior na compreensão de leitura por uma disfunção neurológica, que vai acarretar uma dificuldade de organizar as informações lidas e comprometer diretamente as habilidades necessárias para leitura, dificuldades essas causadas, pois o processo cognitivo linguístico é diretamente alterado ${ }^{8}$.

Um dos recursos que podem ser utilizados para o desenvolvimento da compreensão da leitura é o Mapa Mental. O Mapa Mental foi desenvolvido com o objetivo de facilitar o aprendizado e a memorização, em todos os aspectos da vida. Mais especificamente na Educação, os Mapas Mentais vêm sendo utilizados como uma forma mais didática em sala de aula e até fora dela, pois estimulam a atenção do aluno. Com o Mapa Mental, é possível que o professor prepare sua aula de acordo com a dificuldade de certo grupo, tendo assim maior flexibilidade para apresentar um conteúdo. $\mathrm{O}$ uso do Mapa Mental é indicado também para alunos com dificuldades de aprendizagem, pois é criativo, versátil e utiliza a atenção visual para a sua execução, além de ser um método que apresenta uma facilidade de estabelecer relações entre todas as informações, auxiliando assim o aluno que tem dificuldades nas habilidades de compreensão? .

\section{Objetivo}

O objetivo deste estudo é verificar o índice de mudança e significância clínica do Mapa Mental na intervenção de escolares com transtornos de aprendizagem em situação de pré e pós-testagem.

\section{Material e Método}

Este estudo se caracteriza por ser quase-experimental, realizado com escolares de ensino público municipal da cidade de Marília/SP. Este estudo foi aprovado pelo Comitê de Ética sob o protocolo $\mathrm{n}^{\circ}$ 40514615.8.0000.5406 da Universidade Estadual Paulista - Faculdade de Filosofia e Ciências de Marília "Júlio de Mesquita Filho" (FFC/UNESP).

Participaram deste estudo 7 escolares com diagnóstico interdisciplinar de transtornos de aprendizagem, de ambos os sexos, na faixa etária de 10 anos e 6 meses a 15 anos e 4 meses de idade.

Todos os escolares deste estudo foram submetidos à pré-testagem, intervenção com o mapa mental e pós-testagem, sendo a pré-testagem e a pós-testagem compostas pelo procedimento descrito a seguir:

- Protocolo de Avaliação de Compreensão de Leitura - Procomle ${ }^{\mathbf{1 0}}$ : este estudo utilizou dois textos narrativos ("O Guarda-chuva" e "O Segredo do Armário") desse protocolo, onde cada um é composto por oito perguntas de compreensão de múltipla escolha, sendo quatro relacionadas à microestrutura do texto (duas literais e duas inferenciais) e quatro relacionadas à macroestrutura do texto (duas literais e duas inferenciais). Foi utilizado o texto "O Guarda-chuva" para pré-testagem e "O Segredo do armário" para a pós-testagem.

A aplicação desse procedimento foi realizada individualmente com duração média de 50 minutos em contra turno, no CER/UNESP/FFC (Centro Especializado em Reabilitação) em sala individual de atendimento, sem ruído. 
Após a aplicação dos protocolos em momento de pré-testagem, os escolares foram submetidos à intervenção com o uso do Mapa Mental durante um período de quatro meses. A intervenção foi realizada de forma individual com escolares, semanalmente, com duração de 50 minutos cada sessão.

A intervenção com o uso do Mapa Mental foi dividida em duas etapas; na primeira etapa foi realizada pelo escolar a leitura oral, seguida pela compreensão de um texto seriado; após a leitura, procedeu-se à recontagem da história à pesquisadora, que realizava intervenções quando necessário. Na segunda etapa, o escolar deveria analisar e localizar a ideia central da história, a ordem dos acontecimentos dos fatos e seus fatos mais importantes, criando dessa forma memória textual; realizava, após, a construção de um mapa mental, onde o escolar deveria organizar e escrever as informações em cada estrutura esquemática (título, personagens, ações - desenrolar da história e conclusão - moral da história). Ao todo, foram trabalhados com cada escolar 15 textos.

Os dados de pré e pós-testagem foram analisados por meio do Método $\mathrm{JT}^{11,12}$ para análise de caso único, uma vez que esse método prevê uma análise comparativa entre escores pré e pós-intervenção com o objetivo de estabelecer se as diferenças entre eles representam mudanças confiáveis, e se são clinicamente relevantes, permitindo assim verificar a significância clínica do Mapa Mental.

Esse método é realizado por meio de dois processos complementares: o cálculo da confiabilidade das alterações ocorridas entre a avaliação pré e a avaliação pós-intervenção, descrita em termos de um Índice de Mudança Confiável (IMC); e a análise do significado clínico dessas alterações. A diferença é calculada baseada na diferença entre pré e pós-teste dividida pelo erro-padrão da diferença. Dessa forma, a mudança da pré para a pós-testagem pode ser positiva confiável (quando há melhora); negativa confiável (quando há piora); com significância clínica (que faz ou fará diferença no âmbito clínico); ou ausência de mudança.

É importante salientar que há diferença no efeito de um tratamento de um ponto de vista estatístico para o ponto de vista clínico, independentemente do número de sujeitos ${ }^{13,14}$. Além do mais, há poucos dados na literatura que notificam a necessidade de alterações no método JT; portanto, esse método é eficaz para verificar a significância clínica e controle de mudança comparando o sujeito com ele mesmo ${ }^{14}$.

A análise realizada por meio do método JT gera gráficos, que podem ser transformados em tabelas, a partir dos valores apresentados. Com base no gráfico gerado, podem-se visualizar quatro quadrantes que representam as combinações entre oscilações positivas ou negativas em função da condição inicial e final dos participantes, em que cada ponto ( $\mathrm{Sx}$ ) corresponde a um escolar participante desse estudo.

Como neste estudo a análise de desempenho dos escolares no Protocolo de Avaliação de Compreensão de Leitura foi realizada a partir dos erros, os gráficos se mostrarão ao contrário, pois nesse caso o tipo de indicador é negativo, ou seja, quanto melhor o desempenho do escolar, menor o erro apresentado, portanto, mais baixo o escore.

\section{Resultados}

Foi analisado o desempenho dos escolares deste estudo nas questões literais e inferenciais de textos narrativos do Protocolo de Avaliação de Compreensão de Leitura.

O Gráfico 1 apresenta a confiabilidade de mudança nas questões literais de textos narrativos do Protocolo de Avaliação de Compreensão de Leitura.

Gráfico 1. Confiabilidade de mudança nas questões literais de textos narrativos do Protocolo de Avaliação de Compreensão de Leitura.

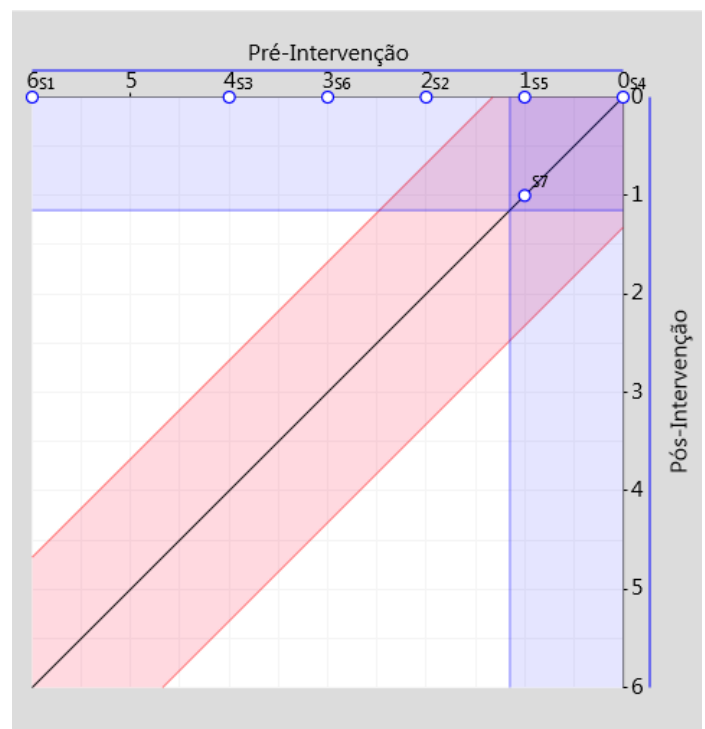


Quanto à significância clínica, os 7 escolares deste estudo estão localizados entre as linhas horizontais superior e inferior em que não se pode afirmar nada sobre mudança de status clínico, pois encontram-se na faixa da incerteza.

Quanto ao índice de mudança confiável, os escolares S1, S2, S3 e S6 estão acima do traçado da diagonal superior, onde apresentaram melhora que pode ser atribuída à intervenção. Os escolares S4, S5 e S7 estão localizados entre as linhas superior e inferior à bissetriz, onde não podem ser feitas afirmações de melhora ou piora devido à intervenção.

O Gráfico 2 apresenta a confiabilidade de mudança nas questões inferenciais de textos narrativos do Protocolo de Avaliação de Compreensão de Leitura.

Gráfico 2. Confiabilidade de mudança nas questões inferenciais de textos narrativos do Protocolo de Avaliação de Compreensão de Leitura.

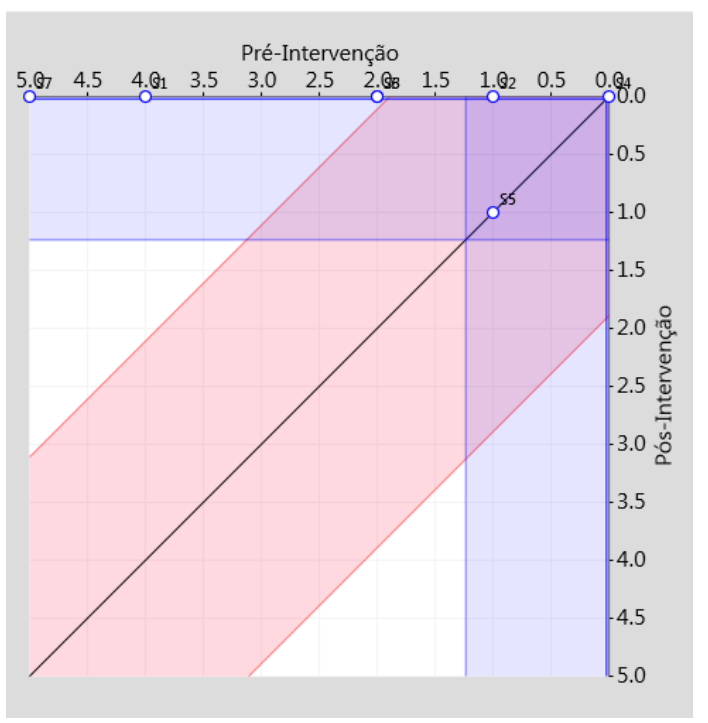

Quanto à Significância Clínica, os escolares S1 a S7 estão localizados entre as linhas horizontais superior e inferior onde não se pode afirmar nada sobre mudança de status clínico, pois encontram-se na faixa da incerteza.

Quanto ao índice de mudança confiável, os escolares S1, S3, S6 e S7 estão acima do traçado da diagonal superior, onde apresentaram melhora que pode ser atribuída à intervenção. Os escolares S2, S4 e S5 estão localizados entre as linhas superior e inferior à bissetriz, onde não podem ser feitas afirmações de melhora ou piora devido à intervenção.

\section{Discussão}

A leitura é considerada efetiva quando há compreensão do material lido. É uma habilidade complexa que envolve vários aspectos cognitivos, como memória de trabalho, conhecimento armazenado, monitoramento, integração de informações e inferências, além de linguísticos, como elementos sintáticos, semânticos, lexicais e decodificação $0^{15}$.

A compreensão da leitura é uma habilidade que exige o emprego de processos considerados básicos, como a decodificação que se refere ao reconhecimento de palavras (processo que permite transformar os signos ortográficos das palavras escritas em linguagem) e extração do significado das palavras impressas, além de ser necessário que o leitor utilize também os processos cognitivos considerados de alto nível, como a capacidade de realizar inferências ${ }^{16}$.

Quando se afirmar que algum escolar tem dificuldade em compreensão de leitura, o avaliador deve fundamentar-se em um teste que seja confiável, pois, para que haja uma boa compreensão de leitura, o leitor deve abstrair ideias do texto, contextualizar e ter estratégias que lhe permitam recorrer aos elementos intra, inter e extratextuais para chegar à compreensão. Sendo assim, foi selecionado para este estudo um instrumento de avaliação pré-testagem e pós-testagem que possibilitou identificar o desempenho dos escolares nesses quesitos descritos como fundamentais segundo a literatura ${ }^{3,17}$.

Embora os escolares deste estudo tenham apresentado um índice de mudança confiável em relação à compreensão de leitura, também houve escolares que não apresentaram mudança, o que sugere que a metodologia de alfabetização que está sendo utilizada para os escolares deste estudo não enfoca o ensino de estratégias para o desenvolvimento da compreensão de leitura em sala de aula ${ }^{18}$.

Embora os escolares deste estudo sejam diagnosticados com transtornos de aprendizagem, esta pesquisa corrobora estudos ${ }^{19,16}$ que sugerem que escolares do $5^{\circ}$ ano empregam o seu desenvolvimento da memória de trabalho e das experiências com textos e se apoiam na memória para as questões serem respondidas.

De acordo com Macedo e colaboradores ${ }^{20}$, experiências de leitura em voz alta e compartilhada, que foi realizada previamente à elaboração do esquema do Mapa Mental, são eficientes para desen- 
volver habilidades prévias para o desenvolvimento da compreensão leitora, bem como a capacidade de fazer inferências, habilidades de memória e o conhecimento de mundo.

Segundo estudos ${ }^{21,22}$, a competência em leitura é a capacidade de o leitor criar suas estratégias para compreensão do material lido, adequando-se ao texto, construindo significado, identificando a macroestrutura, a microestrutura e a superestrutura do texto, estabelecendo assim uma rede de relações e organizando as informações que compõem as diferentes partes do material, realizando inferências, localizando informações relevantes. Sendo assim, conforme descrito neste estudo, a técnica do Mapa Mental é uma técnica relevante e importante para compreensão do texto lido, uma vez que os escolares demonstraram melhora em questões de microestrutura e macroestrutura.

O Mapa Mental é uma técnica relevante para desenvolvimento da compreensão leitora, pois, de acordo com Cunha e Capellini ${ }^{10}$, os escolares tendem a construir um modelo mental do que leem, que é atualizado constantemente de acordo com as relações que estabelecem durante o texto, ou seja, o Mapa Mental de forma esquematizada e escrita possibilita que o escolar concretize as ideias sobre o que leu e compreendeu quando as escreve.

Pode-se afirmar, também, que os escolares deste estudo apresentaram melhora na compreensão leitora, pois a macroestrutura consiste em construir ideias globais que dão sentido e unidade ao texto; portanto, esses escolares conseguiram em situação de pós-testagem realizar a leitura e construir mentalmente ideias globais sobre aquele texto ${ }^{10}$.

Ainda são escassos os estudos descritos na literatura que utilizaram o Mapa Mental como estratégia de intervenção; contudo, este estudo mostrou que a utilização dessa estratégia apresenta um índice de mudança confiável no desempenho de escolares com transtornos de aprendizagem e pode auxiliar no desenvolvimento da compreensão leitora desses escolares.

\section{Conclusão}

Concluímos que a intervenção com o uso do Mapa Mental mostrou-se índice de mudança positiva confiável para escolares com Transtornos de Aprendizagem, podendo ser utilizado como um instrumento de intervenção baseada em evidência científica que auxilie na compreensão da macroestrutura textual, levando o escolar a um bom desempenho nas habilidades de compreensão leitora em textos narrativos.

\section{Referências bibliográficas}

1. Silva C, Capellini S. Eficácia do programa de remediação fonológica e leitura no distúrbio de aprendizagem. Pró-Fono R Atual Cient. 2010; 22(2): 131-38.

2. Silver C, Ruff R, Iverson G, Barth J, Broshek D, Bush S et al. Learning disabilities: The need for neuropsychological evaluation. Arch Clin Neuropsychol. 2008; 23(2): 217-19.

3. Oliveira K, Santos A, Boruchovitch E. A técnica de Cloze na avaliação da compreensão em leitura. In: Organizador: Santos AAA, Boruchovitch E, Oliveira KL., organizadores. Cloze: um instrumento de diagnóstico e intervenção. Pearson; 2009. p. 47-77.

4. Andrade M, Dias M. Processos que levam à compreensão de textos. Psicol Estud. 2006; 11(1): 147-54.

5. Baleghizade S, Babapour M. The effect of summary writing on reading comprehension and recall of EFL students. New England Reading Association J. 2011; 47(1): 44-9.

6. Cain K, Oakhill J, Barnes M, Bryant P. Comprehension skill, inference-making ability, and their relation to knowledge. Mem Cognit. 2001; 29(6): 850-59.

7. Cunha V, Capellini S. Habilidades metalinguísticas no processo de alfabetização de escolares com transtornos de aprendizagem. Rev. Psicopedag. 2011; 28(85): 85-96.

8. Silva C, Capellini S. Desempenho de escolares com e sem transtorno de aprendizagem em leitura, escrita, consciência fonológica, velocidade de processamento e memória de trabalho fonológica. Rev Psicopedag. 2013; 30 (91): 3-11.

9. Buzan T. Speed reading book: the revolutionary approach to increasing reading speed, comprehension and general knowledge (Mind Set). BBC Active; 2006.

10. Cunha V, Capellini S. PROCOMLE: protocolo de avaliação da compreensão de leitura para escolares do $3^{\circ}$ ao $5^{\circ}$ ano. Ribeirão Preto: BookToy; 2014.

11. Jacobson N, Truax P. Clinical significance: A statistical approach to defining meaningful change in psychotherapy research. J Consult Clin Psychol. 1991; 59(1): 12-9.

12. Del Prette Z, Del Prette A. Significância clínica e mudança confiável na avaliação de intervenções psicológicas. Psic: Teor e Pesq. 2008; 24(4): 497-505.

13. Maronesi L, Figueiredo M, Santos E, Mazer-Gonçalves S. Análise de uma intervenção dirigida ao desenvolvimento da coordenação motora fina, global e do equilíbrio. Cad Ter Ocup UFSCar. 2015; 23(2): 273-84.

14. Wise E. Statistical significance testing and clinical effectiveness studies. Psychother. 2011; 48(3): 225-28.

15. Cavalcante T, Leitão S. A natureza argumentativa dos processos inferenciais preditivos na compreensão textual. Estud Psicol (Natal). 2012; 17(1): 35-42.

16. Cunha V, Capellini S. Caracterização do desempenho de escolares do $3^{\circ}$ ao $5^{\circ}$ ano do ensino fundamental em compreensão de leitura. Rev CEFAC. 2016; 18(4): 941-51. 
17. Santos A, Oliveira E. Avaliação e desenvolvimento da compreensão em leitura no ensino fundamental. Psico-USF. 2010; 15(1): 81-91.

18. Uvo M, Germano G, Capellini S. Desempenho de escolares com transtorno do déficit de atenção com hiperatividade em habilidades metalinguísticas, leitura e compreensão leitora. Rev CEFAC. 2017; 19(1): 7-19.

19. Lee Swanson H. Dynamic Testing, Working Memory, and Reading Comprehension Growth in Children With Reading Disabilities. J Learn Disabil. 2011; 44(4): 358-71.

20. Macedo A, et al. Programa fonoaudiológico de promoção de letramento (PFPL): eficácia na compreensão de leitura em escolares. Distúrbios da Comunicação. 2015; 27(2): 248-55.

21. Vicentelli H. Problemática de la lectura en estudiantes universitarios. Psicol Esc Educ. 1999; 3(3): 195-202.

22. Boruchovitch E. Algumas estratégias de compreensão em leitura de alunos do ensino fundamental. Psicol Esc Educ. 2001; 5(1): 19-25. 